\title{
Quantum rings as electron spin beam splitters
}

\author{
Péter Földi, ${ }^{1}$ Orsolya Kálmán, ${ }^{1}$ Mihály G. Benedict, ${ }^{1, *}$ and F. M. Peeters ${ }^{2}$, 团 \\ ${ }^{1}$ Department of Theoretical Physics, University of Szeged, Tisza Lajos körút 84, H-6720 Szeged, Hungary \\ ${ }^{2}$ Departement Fysica, Universiteit Antwerpen, Groenenborgerlaan 171, B-2020 Antwerpen, Belgium
}

\begin{abstract}
Quantum interference and spin-orbit interaction in a one-dimensional mesoscopic semiconductor ring with one input and two output leads can act as a spin beam splitter. Different polarization can be achieved in the two output channels from an originally totally unpolarized incoming spin state, very much like in a Stern-Gerlach apparatus. We determine the relevant parameters such that the device has unit efficiency.
\end{abstract}

PACS numbers: 03.65.-w, 85.35.Ds, 72.25.Dc

The Stern Gerlach experiment, where spatial and spin degrees of freedom become intertwined, has been playing a fundamental role in the conceptual foundations of Quantum Mechanics. Still, soon after the discovery of this effect, it was pointed out by Bohr and Mott $\underline{\underline{1}}$ that, in contrast to atoms, electrons can not be spin-polarized in an inhomogeneous magnetic field. The recent spectacular development of spin electronics (spintronics) ${ }^{2}$ in low dimensional semiconductor structures offers a new way of manipulating spin degrees of freedom. Quantum rings made of semiconducting material ${ }^{\underline{3}}$ exhibiting Rashbatype $e^{\frac{4}{2}}$ spin-orbit interaction (SOI) have been shown to be especially important due to their remarkable spin transformation properties 5.6.7.8.9.10.11.

In the present paper we propose a device that can be considered to a large extent a spintronic analogue of the Stern-Gerlach apparatus: the incoming electrons are forced to split into two different spatial parts by the geometrical construction of the semiconductor device, see Fig. 10 Due to spin-sensitive quantum interference ${ }^{12,13,14}$ and spin-orbit interaction, electrons that are initially in a totally unpolarized spin state become polarized at the outputs with different spin directions. A similar polarizing effect has been predicted in a Y-shaped conductor as a consequence of scattering on impurities ${ }^{15}$ (which is a different physical mechanism from the coherent spin transfer to be discussed here) or because of the presence of SOI in a localized area around the junction 16 There are important proposals considering four terminal devices 17.18 as well, where the strength of the SOI is assumed to be different in the two arms of the interferometer. In our model SOI is uniform in the ring and absent in the leads. However, the latter requirement is not crucial, its purpose is to demonstrate clearly the role of the ring itself, while the effects caused by SOI in the leads can be included in a straightforward way. As our treatment is based on an exact, analytic solution of the spin dependent transport problem, it allows us to determine for which parameters the device is reflectionless, i.e, perfect polarization at the outputs takes place without losses.

We consider a ring ${ }^{19}$ of radius $a$ in the $x-y$ plane and assume a tunable static electric field in the $z$ direction controlling the strength of the spin-orbit interaction characterized by the parameter $\alpha{ }^{\underline{5}}$ The Hamiltoniann ${ }^{7.20}$ in the presence of spin-orbit interaction for a charged particle of effective mass $m^{*}$ is given by

$$
H=\hbar \Omega\left[\left(-i \frac{\partial}{\partial \varphi}+\frac{\omega}{2 \Omega}\left(\sigma_{x} \cos \varphi+\sigma_{y} \sin \varphi\right)\right)^{2}-\frac{\omega^{2}}{4 \Omega^{2}}\right],
$$

where $\varphi$ is the azimuthal angle of a point on the ring, $\hbar \Omega=\hbar^{2} / 2 m^{*} a^{2}$ is the dimensionless kinetic energy of the charged particle and $\omega=\alpha / \hbar a$ is the frequency associated with the SOI. According to Ref. [9], in the $|\uparrow\rangle,|\downarrow\rangle$ eigenbasis of the $z$ component of the spin, the eigenstates of $H$ read:

$$
\psi(\kappa, \varphi)=e^{i \kappa \varphi}\left(\begin{array}{c}
e^{-i \varphi / 2} u(\kappa) \\
e^{i \varphi / 2} v(\kappa)
\end{array}\right) .
$$

The corresponding energy eigenvalues are

$$
E=\hbar \Omega\left[\kappa^{2}-\mu \kappa w+1 / 4\right], \quad \mu= \pm 1,
$$

with $w=\sqrt{1+\left(\omega^{2} / \Omega^{2}\right)}$. The spinors in (2) are simultaneous eigenvectors of $H$, of the $z$ component of the total angular momentum: $K=L_{z}+S_{z}$, and of the spin operator pointing in the direction determined by the angles $\theta$ and $\varphi$ :

$$
S_{\theta \varphi}=S_{x} \sin \theta \cos \varphi+S_{y} \sin \theta \sin \varphi+S_{z} \cos \theta,
$$

where $\theta$ is given by the constant $\tan \theta=-\omega / \Omega$ :

$$
K \psi(\kappa, \varphi)=\kappa_{j}^{\mu} \psi\left(\kappa_{j}^{\mu}, \varphi\right), \quad S_{\theta \varphi} \psi\left(\kappa_{j}^{\mu}, \varphi\right)=\frac{\mu}{2} \psi\left(\kappa_{j}^{\mu}, \varphi\right) .
$$

From geometrical point of view, the second eigenvalue equation above means that the direction of the spinors (2) are either parallel or antiparallel with the conserved (position dependent) direction defined by $S_{\theta \varphi}$. Therefore, the expectation value of the vector $\vec{S}$ in these states rotates around the $z$ direction making always an angle $\theta$ with it, while $\varphi$ is the actual azimuth along the ring.

In a closed ring $\kappa \pm 1 / 2$ should be integer, but the presence of the leads connected to the ring lifts this restriction: the energy is a continuous variable, and then the possible values of $\kappa$ are the solutions of Eq. (3):

$$
\kappa_{j}^{\mu}=\mu\left(w / 2+(-1)^{j} q\right), \quad j=1,2, \quad \mu= \pm 1,
$$


where $q=\sqrt{(\omega / 2 \Omega)^{2}+E / \hbar \Omega}$. The energy eigenvalues are fourfold degenerate, they can be classified ${ }^{9}$ by the quantum numbers $\kappa$ and $\mu$. The ratio of the components of the eigenvectors (2) is determined by $v\left(\kappa_{j}^{\mu}\right) / u\left(\kappa_{j}^{\mu}\right)=$ $(\tan \theta / 2)_{\mu}=\Omega / \omega(1-\mu w)$.

The stationary states of the complete problem including the ring as well as the leads, can be obtained by fitting the solutions corresponding to the different domains. Using local coordinates as shown in Fig. 10 the incoming wave, $\Psi_{3}\left(x_{3}\right)$, and the outgoing waves $\Psi_{1}\left(x_{1}\right), \Psi_{2}\left(x_{2}\right)$ are built up as linear combinations of spinors with spatial dependence $e^{i k x}$ etc. corresponding to $E=\hbar^{2} k^{2} / 2 m^{*}$ :

$$
\begin{aligned}
& \Psi_{3}\left(x_{3}\right)=\left(\begin{array}{c}
f_{\uparrow} \\
f_{\downarrow}
\end{array}\right) e^{i k x_{3}}+\left(\begin{array}{c}
r_{\uparrow} \\
r_{\downarrow}
\end{array}\right) e^{-i k x_{3}}, \\
& \Psi_{n}\left(x_{n}\right)=\left(\begin{array}{c}
t_{\uparrow}^{n} \\
t_{\downarrow}^{n}
\end{array}\right) e^{i k x_{n}},
\end{aligned}
$$

where $n=1,2$. The wave functions belonging to the same

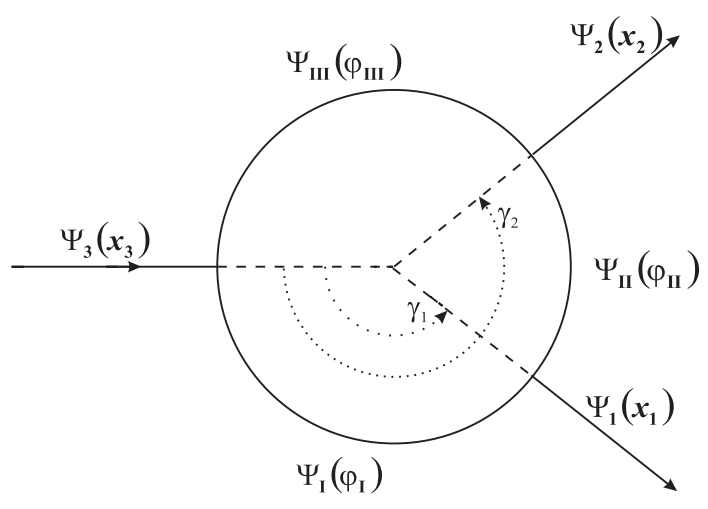

FIG. 1: The geometry of the device and the relevant wave functions in the different domains.

energy $E$ in all the three sections of the ring can be written as linear combinations of four eigenspinors:

$$
\Psi_{i}\left(\varphi_{i}\right)=\sum_{\substack{j=1,2 \\ \mu= \pm 1}} a_{i j \mu} \psi\left(\kappa_{j}^{\mu}, \varphi_{i}\right),
$$

with $i=I, I I, I I I$ identifying the sections. This superposition is no more an eigenvector of $S_{\theta \varphi}$, as it contains states with both $\mu= \pm 1$, and their coefficients are different in general. Additionally, spatial interference of terms describing clockwise and anticlockwise motion plays an essential role in determining the spin direction in the ring. Therefore the position dependencies of the corresponding spin expectation values $\left\langle\Psi_{i}|\vec{S}| \Psi_{i}\right\rangle$ in the arms are more complicated than the simple precession of the eigenvectors given in Eq. (2). Nevertheless, this change of the spin direction along the ring can be calculated without difficulty, if one determines the values of the coefficients $a_{i j \mu}$, which can be done using the boundary conditions, to be discussed now.

Figure 1indicates the wave functions to be fitted at different junctions: e.g. the incoming wave at $x_{3}=0$ should be fitted to $\Psi_{I}$ at $\varphi_{I}=0$ and to $\Psi_{I I I}$ at $\varphi_{I I I}=2 \pi$. We require the continuity of the wave functions, as well as a vanishing spin current density at the junctions $\frac{7.9 .21 .22}{2}$ The procedure is similar to the case of a single outgoing lead which was described in detail in Refs. 7 9]. The results can be summarized by the aid of two transmission matrices which acting on the incoming spinor valued input wave functions provide the output:

$$
T^{(n)}\left(\begin{array}{c}
f_{\uparrow} \\
f_{\downarrow}
\end{array}\right)=\left(\begin{array}{c}
t_{\uparrow}^{n} \\
t_{\downarrow}^{n}
\end{array}\right),
$$

with $n=1,2$. When the incoming electron is not perfectly spin-polarized, its state should be described by a $2 \times 2$ density matrix $\rho_{i n}$, we can write:

$$
\rho^{n}=T^{(n)} \rho_{i n}\left(T^{(n)}\right)^{\dagger},
$$

where $\rho^{1}$ and $\rho^{2}$ are the output density matrices in the respective leads. The matrix elements of $T^{(1)}$ and $T^{(2)}$ can be calculated analytically for arbitrary geometry, but we found that the spin polarizing properties of this device are most clearly seen for the case when the outgoing leads are in a symmetric position, i.e., $\gamma_{1}=2 \pi-\gamma_{2}$. Here we will limit ourselves to this symmetric geometry, yielding

$$
\begin{aligned}
& T_{\uparrow \uparrow}^{(1)}=\frac{8 q a k}{y} e^{i \frac{\gamma_{2}}{2}}\left[\cos ^{2} \frac{\theta}{2}\left(h_{1}+h_{2}\right)+\sin ^{2} \frac{\theta}{2}\left(h_{1}^{*}-h_{2}^{*}\right)\right], \\
& T_{\uparrow \downarrow}^{(1)}=\frac{8 q a k}{y} e^{i \frac{\gamma_{2}}{2}} \sin \frac{\theta}{2} \cos \frac{\theta}{2}\left[\left(h_{1}+h_{2}\right)-\left(h_{1}^{*}-h_{2}^{*}\right)\right], \\
& T_{\downarrow \downarrow}^{(1)}=\frac{8 q a k}{y} e^{-i \frac{\gamma_{2}}{2}}\left[\sin ^{2} \frac{\theta}{2}\left(h_{1}+h_{2}\right)+\cos ^{2} \frac{\theta}{2}\left(h_{1}^{*}-h_{2}^{*}\right)\right],
\end{aligned}
$$

$T_{\downarrow \uparrow}^{(1)}=e^{-i \gamma_{2}} T_{\downarrow \uparrow}^{(1)}$,

where

$$
\begin{aligned}
h_{1}= & -a k e^{-i \frac{w}{2} \gamma_{2}} e^{i w \pi} \sin \left(q\left(2 \pi-\gamma_{2}\right)\right) \sin \left(2 q\left(\pi-\gamma_{2}\right)\right), \\
h_{2}= & i q e^{-i \frac{w}{2} \gamma_{2}}\left[e^{i w \pi} \sin \left(q \gamma_{2}\right)-\sin \left(q\left(2 \pi-\gamma_{2}\right)\right)\right] \\
y= & i a^{3} k^{3}\left[\sin \left(2 q\left(3 \pi-2 \gamma_{2}\right)\right)-2 \sin \left(2 q\left(\pi-\gamma_{2}\right)\right)\right. \\
& -\sin (2 q \pi)]-2 q a^{2} k^{2}\left[\cos \left(2 q\left(3 \pi-2 \gamma_{2}\right)\right)\right. \\
& \left.+2 \cos \left(2 q\left(\pi-\gamma_{2}\right)\right)\right]+6 q a^{2} k^{2} \cos (2 q \pi) \\
& -12 i q^{2} a k \sin (2 q \pi)+8 q^{3}[\cos (w \pi)+\cos (2 q \pi)] .
\end{aligned}
$$

Similarly, for the second output we obtain $T_{\uparrow \uparrow}^{(2)}=T_{\downarrow \downarrow}^{(1)}$, $T_{\downarrow \downarrow}^{(2)}=T_{\uparrow \uparrow}^{(1)}, T_{\uparrow \downarrow}^{(2)}=-T_{\downarrow \uparrow}^{(1)}$ and $T_{\downarrow \uparrow}^{(2)}=-T_{\uparrow \downarrow}^{(1)}$. This symmetry is related to the chosen geometry $\gamma_{1}=2 \pi-\gamma_{2}$. We note that the reflection matrix can also be calculated using the method described above, it turns out to be diagonal in the $\{|\uparrow\rangle,|\downarrow\rangle\}$ basis. We concentrate here on the transmission properties of the ring and consider reflection as a loss in the efficiency of spin transformation.

The most surprising physical consequence of our three terminal ring is its ability to deliver polarized output beams of electrons. Considering a completely unpolarized input, i.e., $\rho_{i n}$ being proportional to the identity, the outputs will be generally partially polarized that could be 
detected by Faraday rotation experiments ${ }^{13}$ However, we found that properly chosen parameters lead to output polarizations as high as $100 \%$. The relevant output density operators in this case should be projectors (apart from the possible reflective losses):

$$
\frac{1}{2} T^{(n)}\left(T^{(n)}\right)^{\dagger}=\eta_{n}\left|\phi^{n}\right\rangle\left\langle\phi^{n}\right| .
$$

The non-negative numbers $\eta_{1}$ and $\eta_{2}$ measure the efficiency of the polarizing device, i.e., $\eta_{1}+\eta_{2}=1$ means a reflectionless process. Direct calculation shows that, provided Eq. (12) is satisfied, the norms of the two outputs are equal, $\eta_{1}=\eta_{2} \equiv \eta / 2$. Eq. (12) is equivalent to requiring the determinants of $T^{(n)}\left(T^{(n)}\right)^{\dagger}$ to vanish. We found that these determinants are equal, and zero if $h_{1} \pm h_{2}=0$. Using Eqs. (111), these conditions can be formulated as

$$
\begin{aligned}
\cos (w \pi) & =\frac{\sin \left(q \gamma_{2}\right)}{\sin \left(q\left(2 \pi-\gamma_{2}\right)\right)}, \\
\sin (w \pi) & =\mp \frac{a k}{q} \sin \left(2 q\left(\pi-\gamma_{2}\right)\right),
\end{aligned}
$$

each of them lead to a $k-\omega$ relation as depicted in Fig. 2 for a representative example corresponding to $\gamma_{2}=3 \pi / 2$. The crossing points of the gray (solution of Eq. (13a) and black (solution of Eq. (13b) curves in Fig. 2 are the parameters that can be used in an experimental realization of our proposal to achieve perfectly polarized outputs. Similar figures can be drawn for arbitrary (symmetric)

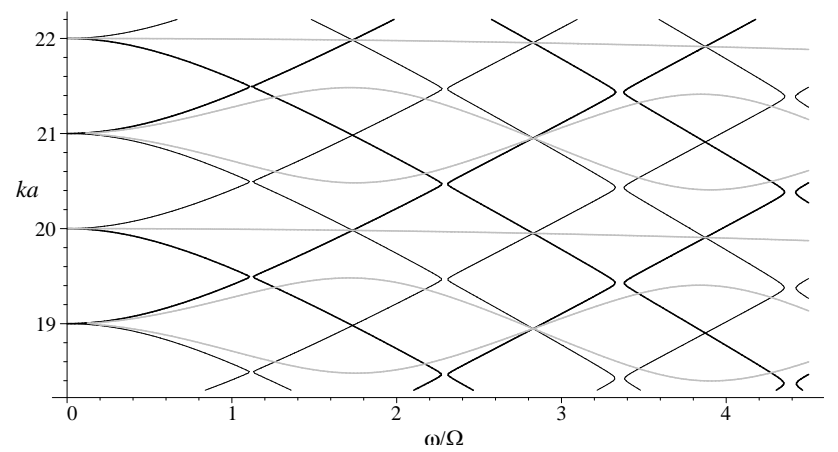

FIG. 2: Determination of the parameter values corresponding to perfect polarization: Eq. 13a and Eq. 13b with the plus (minus) sign are satisfied along the gray and the thin (thick) black lines, respectively. At each intersection of a black and a gray line the ring acts as a perfect polarizing device. This figure corresponds to geometry given by $\gamma_{1}=\pi / 2, \gamma_{2}=3 \pi / 2$.

geometry. This implies that there are lines in three dimensional $\left\{\gamma_{2}, \omega / \Omega, k a\right\}$ space along which the ring polarizes a completely unpolarized input.

Now we can ask what the transmission probabilities are, provided perfect polarization occurs. Fig. 3 shows that along a line defined by $h_{1}+h_{2}=0, \eta$ is a quasiperiodic function of $\gamma_{2}$. A similar figure can be drawn for the condition $h_{1}-h_{2}=0$. As we can see, there are certain points (that is, parameter combinations), where the transmission probability is unity. This shows that it is possible to obtain $100 \%$ spin polarized outputs from a perfectly unpolarized input, even without reflective losses.

Now we turn to the investigation of the outgoing spinors which arise as a consequence of the polarizing property of the ring. Clearly, these are the eigenstates $\left|\phi^{n}\right\rangle$ of the transmitted density matrices corresponding to the nonzero eigenvalues which are given by $\eta_{1}=\eta_{2}=128 q^{2} a^{2} k^{2}\left|h_{1}\right|^{2} /|y|^{2}$. Note that the quasiperiodic behavior of the transmission probability $\eta=\eta_{1}+\eta_{2}$ seen in Fig. [3 is related to the sine and cosine functions in $h_{1}$ and $y$. Focusing on the case of $h_{1}+h_{2}=0$, the eigenstates of the respective transmitted density matrices corresponding to the nonzero eigenvalues $\eta_{1}$ and $\eta_{2}$ read

$$
\left|\phi^{1}\right\rangle_{+}=\left(\begin{array}{c}
\sin \frac{\theta}{2} \\
-e^{-i \gamma_{2}} \cos \frac{\theta}{2}
\end{array}\right), \quad\left|\phi^{2}\right\rangle_{+}=\left(\begin{array}{c}
e^{-i \gamma_{2}} \cos \frac{\theta}{2} \\
\sin \frac{\theta}{2}
\end{array}\right) .
$$

These results describe the connection between the strength of the spin-orbit coupling (encoded in $\theta$ ), the geometry of the device and its polarizing directions. We stress that this pair of spinors exhibits nontrivial spatial-spin correlation being a signature of quantum non-contextuality ${ }^{23}$ However, note that they are in general not orthogonal, their overlap is given by $+\left\langle\phi^{2} \mid \phi^{1}\right\rangle_{+}=$ $i \sin \theta \sin \gamma_{2}$. On the distinguishability of nonorthogonal states, see Ref. 24]. Similarly, for $h_{1}-h_{2}=0$, we have:

$$
\left|\phi^{1}\right\rangle_{-}=\left(\begin{array}{c}
e^{i \gamma_{2}} \cos \frac{\theta}{2} \\
\sin \frac{\theta}{2}
\end{array}\right),\left|\phi^{2}\right\rangle_{-}=\left(\begin{array}{c}
\sin \frac{\theta}{2} \\
-e^{i \gamma_{2}} \cos \frac{\theta}{2}
\end{array}\right)
$$

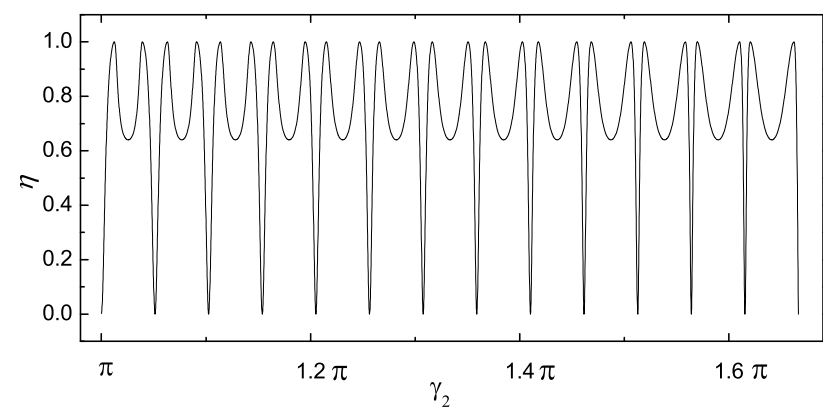

FIG. 3: The transmission probability of a perfectly polarizing ring as a function of $\gamma_{2}=2 \pi-\gamma_{1}$. The parameter $k a$ changes in the range of $[19.0,21.0]$, while $0<\omega / \Omega<5$, and the plot corresponds to the condition $h_{1}+h_{2}=0$.

Considering the transmission matrices themselves, it is clear that under the conditions given by Eqs. [13a b), their determinants also vanish. That is, each $T^{(n)}$ has a zero eigenvalue, but - due to the nonhermiticity - its eigenspinors are not orthogonal. It can be verified that the eigenstates corresponding to the nonzero eigenvalue coincide with $\left|\phi^{n}\right\rangle_{+}$and $\left|\phi^{n}\right\rangle_{-}$, while the spinors annulled by the transmission matrices $T^{(n)}\left|\phi_{0}^{n}\right\rangle=0$ have 
the following components:

$$
\left|\phi_{0}^{1}\right\rangle_{+}=\left(\begin{array}{c}
\cos \frac{\theta}{2} \\
\sin \frac{\theta}{2}
\end{array}\right), \quad\left|\phi_{0}^{2}\right\rangle_{+}=\left(\begin{array}{c}
-\sin \frac{\theta}{2} \\
\cos \frac{\theta}{2}
\end{array}\right)
$$

if $h_{1}+h_{2}=0$, and $\left|\phi_{0}^{1}\right\rangle_{-}=\left|\phi_{0}^{2}\right\rangle_{+},\left|\phi_{0}^{2}\right\rangle_{-}=\left|\phi_{0}^{1}\right\rangle_{+}$.

This shows that if the conditions given by Eqs. (13a-b) are satisfied, the device acts similar to a Stern-Gerlach apparatus in the sense that: 1) for unpolarized input, we have two different spin directions (14) in the outputs, 2) if we consider one of the eigenstates (14) as the input, its spin direction will not change in the appropriate output, and 3) there are spinors given by Eq. (16), for which the transmission probability into a given output lead is zero. However, the analogy is not perfect, the polarized spinors (14) are not orthogonal and the spinor which has zero probability to be transmitted through a given lead is not equal to the eigenstate corresponding to the nonzero eigenvalue of the other lead: $\left|\phi^{n}\right\rangle \neq\left|\phi_{0}^{n^{\prime}}\right\rangle$ for $n \neq n^{\prime}$. From this point of view, an optical polarizing beam splitter ${ }^{25.26}$ with nonorthogonal polarizing directions can be the closest analogue.

The present calculation was done for an idealized model system; in fact, our intention was showing that the discussed polarizing effect - in contrast to previous proposals - can be described in terms of pure Quantum Mechanics (i.e., spin precession and interference), thus it is of importance from a fundamental point of view, as well. On the other hand, there are results showing that the approximations of our model (transport is ballistic and one dimensional, i.e., the finite width of the ring-wire was not taken into account) can give valid description of actual physical systems under specific experimental situations. Currently, high mobility samples have become available such that at cryogenic temperatures transport is found to be ballistic over tens of microns. Similarly, phase coherence and spin coherence lengths ${ }^{27}$ have been found up to $100 \mu \mathrm{m}$. Our narrow ring implies the assumption of single mode propagation. Recently, it was found that the finite width of the rings has a small effect on the loss of coherence of the spin state; it has also been shown that in a multi-channel system the modulation of the transmitted spin states survive and under specific conditions the individual eigenchannel transmissions are very similar to the ones found in single channel rings 8.28 A possible non-ideal coupling to the leads can be described through effective tunnel barriers. But in most of the current experimental systems the leads are connected in a rather adiabatic way which makes the coupling very close to ideal.

In conclusion, we showed that a quantum ring with one input and two output leads in the presence of Rashba-type SOI has remarkable similarities with a Stern-Gerlach apparatus. Parameter values, within the experimentally feasible range ${ }^{5.6 .29}$ were identified when the three terminal ring delivers perfectly polarized output beams of electrons without reflective losses. We found that appropriate spin polarized input states are transmitted without modification, but it is also possible to prepare inputs, for which the transmission into a given lead is forbidden. Thus our paper describes a realistic model in which spin sensitive quantum interference gives rise to fundamental polarization effects as well as to nontrivial spatial-spin correlations.

We note that similar rings can act as spintronic quantum gates 9 or in the presence of an external magnetic field can be used also for spin filtering $\underline{\underline{7}}^{\underline{T}}$ This points to the possibility to integrate spintronic beam splitters, gates and filters that can serve as elementary building blocks of a quantum network based on spin sensitive devices $, 30,31,32,33$

Acknowledgments: This work was supported by the Flemish-Hungarian Bilateral Programme, the Flemish Science Foundation (FWO-Vl), the Belgian Science Policy and the Hungarian Scientific Research Fund (OTKA) under Contracts Nos. T48888, D46043, M36803, M045596.
* Electronic address: benedict@physx.u-szeged.hu

$\dagger$ Electronic address: francois.peeters@ua.ac.be

1 N. F. Mott and H. S. W. Massey, The theory of atomic collissions (Clarendon Press, Oxford, 1949), 2nd ed.

2 I. Žutić, J. Fabian, and S. D. Sarma, Rev. Mod. Phys. 76, 323 (2004).

3 S. Viefers, P. Koskinen, P. S. Deo, and M. Manninen, Physica E 21, 1 (2004).

${ }^{4}$ E. I. Rashba, Sov. Phys. Solid State 2, 1109 (1960).

5 J. Nitta, T. Akazaki, H. Takayanagi, and T. Enoki, Phys. Rev. Lett. 78, 1335 (1997).

6 J. Nitta, F. E. Meijer, and H. Takayanagi, Appl. Phys. Lett. 75, 695 (1999).

7 B. Molnár, F. M. Peeters, and P. Vasilopoulos, Phys. Rev. B 69, 155335 (2004).

8 D. Frustaglia and K. Richter, Phys. Rev. B 69, 235310
(2004)

9 P. Földi, B. Molnár, M. G. Benedict, and F. M. Peeters, Phys. Rev. B 71, 033309 (2005).

10 F. Zhai and H. Q. Xu, Phys. Rev. Lett. 94, 246601 (2005).

11 T. Koga, J. Nitta, and M. van Veenhuizen, Phys. Rev. B 70, 161302(R) (2004).

12 S. Souma and B. Nikolić, Phys. Rev. Lett. 94, 106602 (2005).

13 Y. K. Kato, R. C. Myers, A. C. Gossard, and D. D. Awschalom, Appl. Phys. Lett. 86, 162107 (2005).

14 J. Cserti, A. Csordás, U. Zülicke, Phys. Rev. B 70, 233307 (2004).

15 T. P. Pareek, Phys. Rev. Lett. 92, 076601 (2004).

16 A. A. Kiselev and K. W. Kim, Appl. Phys. Lett. 78, 775 (2001).

17 R. Ionicioiu and I. D'Amico, Phys. Rev. B 67, 041307 (R) 
(2003).

18 M. Governale, D. Boese, U. Zülicke, and C. Schroll, Phys. Rev. B 65, 140403 (R) (2002).

19 A. G. Aronov and Y. B. Lyanda-Geller, Phys. Rev. Lett. 70, 343 (1993).

${ }^{20}$ F. E. Meijer, A. F. Morpurgo, and T. M. Klapwijk, Phys. Rev. B 66, 033107 (2002).

21 S. Griffith, Trans. Faraday Soc. 49, 345 (1953).

22 J. B. Xia, Phys. Rev. B 45, 3593 (1992).

${ }^{23}$ Y. Hasegawa, R. Loidl, G. Badurek, M. Baron, and H. Rauch, Nature 425, 45 (2003).

24 J. A. Bergou, U. Herzog, and M. Hillery, Phys. Rev. Lett. 90, 257901 (2003).

25 R. Loudon, The quantum theory of light (Cambridge Univ. Press, Cambridge, 2000), 3rd ed.

26 J. K. Asbóth, P. Adam, M. Koniorczyk, and J. Janszky,
Eur. Phys. J. D 30, 403410 (2004).

27 J. M. Kikkawa and D. D. Awschalom, Phys. Rev. Lett. 80, 4313 (1998).

28 S. Souma and B. Nikolić, Phys. Rev. B 70, 195346 (2004).

29 Y. Sato, S. G. T. Kita, and S. Yamada, J. Appl. Phys. 89, 8017 (2001).

30 J. C. Euges, G. Burkard, and D. Loss, Appl. Phys. Lett. 82, 2658 (2003).

31 D. Stepanenko, N. E. Bonesteel, D. P. DiVincenzo, G. Burkard, and D. Loss, Appl. Phys. Lett. 68, 115306 (2003).

32 J. B. Yau, E. P. DePoortere, and M. Shayegan, Phys. Rev. Lett. 88, 146801 (2003).

33 D. Frustaglia, M. Hentschel, and K. Richter, Phys. Rev. Lett. 87, 256602 (2001). 